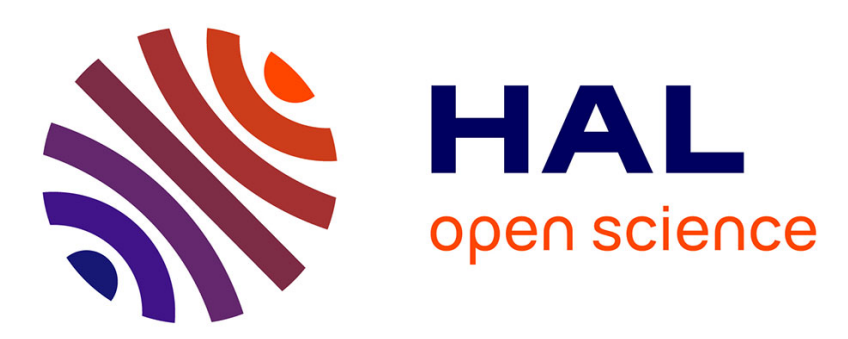

\title{
A college admissions game for uplink user association in wireless small cell networks
}

Walid Saad, Zhu Han, Rong Zheng, Merouane Debbah, Harold Vincent Poor

\section{To cite this version:}

Walid Saad, Zhu Han, Rong Zheng, Merouane Debbah, Harold Vincent Poor. A college admissions game for uplink user association in wireless small cell networks. INFOCOM, 2014 Proceedings IEEE, Apr 2014, Toronto, Canada. 10.1109/INFOCOM.2014.6848040 . hal-01098823

\section{HAL Id: hal-01098823 https://hal.science/hal-01098823}

Submitted on 16 Dec 2015

HAL is a multi-disciplinary open access archive for the deposit and dissemination of scientific research documents, whether they are published or not. The documents may come from teaching and research institutions in France or abroad, or from public or private research centers.
L'archive ouverte pluridisciplinaire HAL, est destinée au dépôt et à la diffusion de documents scientifiques de niveau recherche, publiés ou non, émanant des établissements d'enseignement et de recherche français ou étrangers, des laboratoires publics ou privés. 


\title{
A College Admissions Game for Uplink User Association in Wireless Small Cell Networks
}

\author{
Walid Saad ${ }^{1}$, Zhu Han ${ }^{2}$, Rong Zheng ${ }^{3}$, Mérouane Debbah ${ }^{4}$, and H. Vincent Poor ${ }^{1}$ \\ ${ }^{1}$ Electrical and Computer Engineering Department, University of Miami, USA, walid@miami.edu. \\ ${ }^{2}$ Electrical and Computer Engineering Department, University of Houston, USA, zhan2@uh.edu. \\ ${ }^{3}$ Department of Computing and Software, McMaster University, Hamilton, ON, Canada, email: rzheng@mcmaster.ca. \\ ${ }^{4}$ Alcatel-Lucent Chair in Flexible Radio, Supelec, Gif-sur-Yvette, France email: merouane.debbah@supelec. fr. \\ ${ }^{1}$ Electrical Engineering Department, Princeton University, USA, poor@princeton. edu.
}

\begin{abstract}
In this paper, the problem of uplink user association in small cell networks, which involves interactions between users, small cell base stations, and macro-cell stations, having often conflicting objectives, is considered. The problem is formulated as a college admissions game with transfers in which a number of colleges, i.e., small cell and macro-cell stations seek to recruit a number of students, i.e., users. In this game, the users and access points (small cells and macro-cells) rank one another based on preference functions that capture the users' need to optimize their utilities which are functions of packet success rate (PSR) and delay as well as the small cells' incentive to extend the macro-cell coverage (e.g., via cell biasing/range expansion) while maintaining the users' quality-of-service. A distributed algorithm that combines notions from matching theory and coalitional games is proposed to solve the game. The convergence of the algorithm is shown and the properties of the resulting assignments are discussed. Simulation results show that the proposed approach yields a performance improvement, in terms of the average utility per user, reaching up to $23 \%$ relative to a conventional, best-PSR algorithm.
\end{abstract}

\section{INTRODUCTION}

Meeting the stringent quality-of-service (QoS) requirements of emerging wireless services warrants substantial changes in current cellular infrastructure. In this respect, the introduction of small cell base stations (SCBSs) (picocells, microcells, femtocells, etc.) is seen as a promising, cost-effective solution [1-3]. Small cells are low-cost, low-power, access points that can be overlaid on any existing wireless technology (e.g., 2G, 3G, LTE, WiMAX, etc.) and can be of different types that include outdoor, operator-deployed picocells, metrocells and microcells, as well as indoor, user-deployed femtocells [1]. These SCBSs are seen as a key approach for offloading traffic from the macro-cell network while providing high capacities by bringing the users and their access points closer to one another [2].

For reaping the benefits of small cell deployment, numerous technical challenges must be addressed such as resource management, interference avoidance, and user association [2], [4-9]. In [5], the authors formulate a hierarchical power control game between the macro-cellular base station and the small cells so as to reduce the interference. The authors in [10] study the problem of cell range expansion in picocell networks and derive fundamental results. Further, a novel approach for spectrum allocation in two-tier networks is proposed in [11]. In [6], a new approach for resource allocation in heterogeneous networks is developed via auctions. Other aspects of small cell networks are studied in [7-9]

One key challenge in small cell networks that remains relatively unexplored, as outlined in [1] and [12] (and references therein), is the problem of associating the users to their serving access point for uplink transmission, in particular. Inherently,

This research was supported by the U.S. National Science Foundation under Grants CNS-1253731, CNS-1265268, CNS-1117560, ECCS-1028782, and CNS-0953377. within a small cell network, the problem of user association in the uplink faces a number of pertinent challenges that are significantly different from classical cellular user association problems such as those in [13] and [14]. On the one hand, unlike macro-cell base stations, SCBSs are resource-constrained in nature (e.g., can service only a small number of users and have limited coverage) which can significantly impact the user association problem. On the other hand, within a small cell network, the outcome of any access point selection process must account for the preferences of three node types (and not just the users as in cellular networks): the users, the SCBSs, and the macro-cell stations, having different, often conflicting, goals. From the users' perspective, the goal is primarily to select a serving station, irrespective of its type (macro-cell or SCBS), that optimizes a certain QoS requirement. For the SCBSs, the objective is not only to select the users with certain desired QoS requirements, but also to improve the coverage of the macro-cell network and alleviate the load on its stations. Offloading data is, in fact, viewed as the primary role of SCBSs, particularly those deployed by an operator in outdoor environments such as picocells [1], [2], [15]. Finally, the macro-cell stations aim at improving the QoS of their users while ensuring that every user gets a chance to transmit. Often, these objectives are intertwined and can lead to conflicting user assignment choices. For example, the most preferred access point of a certain user might be interested in assigning other users that are more aligned with its own utility metrics (e.g., allow a better offloading) and vice versa. These new challenges motivate the design of novel approaches for user-to-access point assignment tailored to small cell networks, which is our main focus. In fact, it has been recently shown [12] that, in the downlink, applying classical macro-cell oriented schemes to small cell networks can lead to highly unequal loads and operating inefficiencies. The work in [12] developed an optimization problem for maximizing capacity during user association in the downlink of small cell networks. To our best knowledge, beyond [12], which is focused on downlink problem and on centralized optimization, little work has been done to address the SCBSs user to access point association problem in the uplink while accounting for the aforementioned challenges.

The main contributions of this paper are to study and design, using analytical techniques from game theory, novel strategies for assigning users to their serving access points within the uplink of a small cell network. Here, we exploit the striking analogy between the small cells' user association problem and the college admissions game that is studied in the game theory literature. We formulate a college admissions game in which colleges, i.e., access points (SCBSs and macro-cells) that have a fixed quota (number of students to admit) and students, i.e., net- 
work users, rank one another based on preference functions that capture, for the users, the R-factor which is a well-known metric capturing the packet success rate and delay guarantees and, for the access points, the need to service users with good quality of service while extending the macro-cell coverage (e.g., similar to the emerging idea of biasing or cell range expansion [1], [12], [15]). To solve this game, we propose an algorithm that takes into account the interdependent users' preferences and that allows each node to optimize its specific utility needs. Using analytical techniques based on the college admissions game and coalitional game theory, we provide a novel formulation for the problem of access point selection tailored to the unique features of small cell wireless networks (e.g., different decision makers, conflicting goals, limited SCBS capacity, etc.). We show that, using the proposed approach, the users, SCBSs, and macro-cells can agree on a user association outcome, given their different preferences. We show the convergence of the algorithm and we analyze the properties of the resulting assignment. We assess the performance of the algorithm using simulations and show that it yields significant performance gains for all nodes.

The rest of this paper is organized as follows. In Section II, we present the proposed system model. In Section III, we formulate the problem as a college admissions game with transfers while in Section IV we propose an algorithm for network assignment. Simulation results are presented in Section V. Finally, conclusions are drawn in Section VI.

\section{System MOdEL}

\section{A. Network Model}

Consider a wireless network composed of $M$ cells with their associated base stations, i.e., macro-stations. $N$ wireless users are deployed and seek to transmit in the uplink direction. Hereinafter, we refer to this main network as the macro-cell network. $K$ SCBSs are overlaid on the macro-cell network to increase coverage and improve the performance of the users. We let $\mathcal{M}, \mathcal{K}$, and $\mathcal{N}$, denote, respectively, the set of all macro-cell base stations, all SCBSs, and all users.

Each SCBS $k \in \mathcal{K}$ is a low-cost access point that can serve a limited number of users, i.e., has a maximum quota of $q_{k}$ users. We mainly focus on outdoor SCBSs such as picocells/microcells/metrocells that are used by the operator to offload data by serving any of the $N$ macro-cell users when needed [1], [2]. We note that the quota of an SCBS can be either fixed or controlled by the SCBS operator who may decide to allow less or more users to connect [1]. Further, we consider that all SCBSs and macro-stations use a time-division multipleaccess scheduler with each time slot having a duration $\theta$. Note that, using such a transmission for the uplink implies that the users which are assigned to the same SCBS or macro-station do not interfere with one another, i.e., there no intra-access point (SCBS or macro-station) interference. Note that the analysis done in this paper is equally applicable to other multiple access and scheduling schemes, e.g., advanced QoS-aware schedulers such as those in [1] and references therein. For brevity, we use the term access point to refer to a macro-station or an SCBS if the explicit distinction is not needed.

A key problem in small cell networks is to associate the users to their serving access points which are either macro-stations or SCBSs [1]. An illustrative figure of this problem is shown in Fig. 1 for a network with $M=3$ macro-stations, $K=3$ SCBSs,

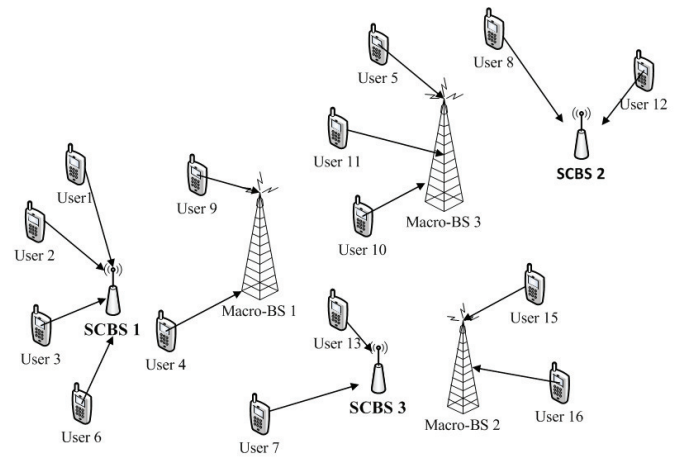

College admissions game involving three decision makers - Users (students) that need to improve their performance

SCBSs with limited quota (Universities) that need to ensure users' QoS and help offload traffic from the macro-cell -Macro-BSs (Universities) that need to ensure that all users are properly serviced

Fig. 1. An illustration of a small cell network network with $M=3$ macrostations, $K=3 \mathrm{SCBSs}$, and $N=16$ users.

and $N=16$ users. Fig. 1 shows how the SCBSs can extend the coverage of the macro-stations, notably, SCBSs 2 and 3 enable servicing users that are far away from macro-stations 3 (e.g., user 12) and 1 (e.g., user 7), respectively. At SCBS 1, which can service at most 4 users, we can see how the different objectives of the nodes and the limitations of the SCBSs impact the user assignment. For example, although user 4 prefers to connect to SCBS 1 over macro-station 1 due to proximity, this user is eventually assigned to macro-station 1 . This assignment is a result of the fact that SCBS 1 needs to also assist in offloading traffic from the macro-cell network by servicing users that are further away from the macro-stations, e.g., users 1,2,3, and 6 . Also, Fig. 1 shows how some users, such as user 8, prefer lightly loaded stations such as SCBS 2 over closer, yet congested access points such as macro-station 1 . Thus, Fig. 1 sheds light on the challenges of designing assignment schemes in the uplink of small cell networks.

\section{B. Objectives of the Nodes}

Users' goals: In the uplink, each user $i \in \mathcal{N}$ chooses an access point $a \in \mathcal{A} \triangleq \mathcal{M} \cup \mathcal{K}$, and, thus, achieves a signalto-interference plus noise ratio $\gamma_{i a}$ and perceives a delay $\tau_{a}$. Each user $i \in \mathcal{N}$ wants to select an access point $a \in \mathcal{A}$ so as to optimize its probability of successful transmission and its perceived delay. The packet success rate (PSR) $\rho_{i a}$ of any user $i \in \mathcal{N}$ that needs to transmits packets of $B$ bits each to an access point $a \in \mathcal{A}$ is

$$
\rho_{i a}=\left(1-P_{i a}^{\mathrm{e}}\left(\gamma_{i a}\right)\right)^{B},
$$

where $P_{i a}^{\mathrm{e}}\left(\gamma_{i a}\right)$ is the bit error rate (BER) from user $i$ to its serving station $a$ and is a function of $\gamma_{i a}=\frac{P_{i} g_{i a}}{\sigma^{2}+I_{a}}$ with $P_{i}$ being the transmit power of user $i, \sigma^{2}$ the noise variance, $I_{a}$ the inter-access point interference temperature ${ }^{1}$ at $a$, and $g_{i a}=\frac{1}{d_{i a}^{\alpha}}$ the channel gain between user $i$ and access point $a$ with $d_{i a}^{i a}$ the distance between $i$ and $a$, and $\alpha$ the path loss exponent ${ }^{2}$. Essentially, the expression for the BER depends on the coding and modulation schemes adopted by user $i$. The analysis done in the remainder of this paper is applicable to any coding and modulation scheme. Note that, in practice, interference

${ }^{1} I_{a}$ is a measure of the average interference temperature at $a$ incurred from potential uplink transmissions at other access points that can interfere with $a$ (e.g., share the spectrum with $a$ ) that is commonly used in dimensioning small cell networks [1].

${ }^{2}$ We note that the choice of this channel model is made for convenience and without loss of generality since the proposed game-theoretic approach is also applicable when the channel gain encompasses other propagation characteristics such as shadowing effects and multi-path fading. 
between SCBSs is often considered to be well-managed (e.g., by allowing the SCBSs to perform sensing so as to avoid using the same part of the spectrum or using other methods) such as in [4], [16], [17]. Hence, $I_{a}$ is mostly a measure of the macroto-femto interference temperature which can also be improved or canceled by having a macro-cell that uses a dedicated band such as in [1] or using techniques such as in [16]. Hereafter, we consider a slowly varying block fading channel model with a long coherence time that is constant over the slot duration $\theta$.

Given an access point $a \in \mathcal{A}$ that serves a subset of users $\mathcal{N}_{a} \subseteq \mathcal{N}$, each user $i \in \mathcal{N}_{a}$ experiences a delay $\tau_{a}$ that is mainly dominated by two components, a wireless access delay $\tau_{a}^{\mathrm{W}}$ and a backhaul delay $\tau_{a}^{\mathrm{b}}$, such that

$$
\tau_{a}=\tau_{a}^{\mathrm{w}}+\tau_{a}^{\mathrm{b}} .
$$

The wireless access delay $\tau_{a}^{\mathrm{w}}$ depends primarily on the number of users connected to $a$, i.e., $N_{a}=\left|\mathcal{N}_{a}\right|$ where $|\cdot|$ denotes the set cardinality. Given the considered users' scheduler, we have

$$
\tau_{a}^{\mathrm{w}}=\left(N_{a}-1\right) \cdot \theta
$$

where $\theta$ is the slot duration. The delay over the backhaul $\tau_{a}^{\mathrm{b}}$ at an access point $a$ depends on several factors such as the capability of the backbone, its connectivity (e.g., to the Internet), the backbone topology, as well as the traffic which is generated, not only by the users in $\mathcal{N}_{a}$ but also from third-party applications or service providers. Hence, providing accurate models for the delay over the backhaul network is a challenging research problem that is solved often using empirical measurements [18] and is beyond the scope of this paper. Since our focus is mainly on the user association problem, we consider that the delay $\tau_{a}^{\mathrm{b}}$ over the backhaul during a given time period is a random variable having a certain observed distribution. Due to the technical differences between the backbone connecting the macro-stations (which can be a high-speed network, e.g., fiber) and the one connecting the SCBSs (which is a standard IP backaul, e.g, DSL), we distinguish between the characteristics of the distribution of the backhaul delay at a macro-cell, i.e., $\tau_{m}^{\mathrm{b}}, m \in \mathcal{M}$ and at a small cell, i.e., $\tau_{k}^{\mathrm{b}}, k \in \mathcal{K}$.

Each user $i \in \mathcal{N}$ selects an access point $a \in \mathcal{A}$ so as to optimize its PSR in (1) and its delay in (2). One suitable metric that captures both PSR and delay is the R-factor which is popular within voice over IP (VoIP) services [19]. For a user $i$, the R-factor is an expression that links the delay and packet loss to the voice quality as follows:

$$
\begin{array}{r}
U_{i}\left(\rho_{i a}, \tau_{a}\right)=\Omega-\epsilon_{1} \tau_{a}-\epsilon_{2}\left(\tau_{a}-\alpha_{3}\right) H-v_{1} \\
-v_{2} \ln \left(1+100 v_{3}\left(1-\rho_{i a}\right)\right),
\end{array}
$$

where $\tau_{a}$ is the delay given by (2) expressed in milliseconds, and $100\left(1-\rho_{i a}\right)$ represents the packet loss percentage. The remaining parameters are constants defined as: $\Omega=94.2, \epsilon_{1}=$ $0.024, \epsilon_{2}=0.11, \epsilon_{3}=177.3, H=0$ if $\tau_{j}<\epsilon_{3}, H=1$ otherwise [19]. The parameters $v_{1}, v_{2}$, and $v_{3}$ depend on the codec as per the e-model [19]. The relationship between the Rfactor and the VoIP service quality is such that as the R-factor increases, by increments of 10 , from 50 to 100 , the voice quality is poor, acceptable, good, high, and best, respectively [19].

SCBSs and macro-stations goals: The SCBSs have two objectives: 1) to offload traffic from the macro-cell, extend its coverage, and load balance the traffic (e.g., use cell range expansion or biasing) and, 2) to select users that can potentially experience a good R-factor. For exploiting the full potential of SCBS deployments, it is desirable to actively "push" wireless users onto the SCBSs while maintaining a good QoS, when possible due to two reason: (i) the SCBSs will often be lightly loaded, thus a better load balancing strategy is required; and (ii) this allows offloading of traffic and reducing the load on the main macro-cell network. To assist the macro-cell by extending its coverage and offloading users, an SCBS $k$ benefits from selecting the users in poor macro-cell coverage areas, i.e., users that experience the largest packet drop at the macro-stations (e.g., far from these stations). However, at the same time, an SCBS $k$ also extracts more utility by selecting users that can potentially experience a good QoS when connected to $k$. Many techniques have recently been studied to allow efficient offload. In particular, the idea of cell biasing or cell range expansion which has been recently proposed suggests forcing users to maintain their connections at smaller tiers (e.g., at SCBSs) by "biasing" their transmit power with a multiplicative factor [12], [20].

From the perspective of an SCBS, the dominating indicator on the R-factor of a given user $i \in \mathcal{N}$ is the PSR as per (1). Therefore, in general, the benefit (utility) that any SCBS $k \in \mathcal{K}$ obtains by serving a user $i \in \mathcal{N}$, can be written as

$$
h_{k}(i)=f\left(\rho_{i k}, \rho_{i m}\right) \text {, }
$$

where $\rho_{i k}$ is the PSR from user $i$ to $\operatorname{SCBS} k, \rho_{i m}$ is the PSR from user $i$ to its best macro-station $m$, i.e., $m \in$ $\arg \max _{l \in \mathcal{M}} \rho_{i l}$, and $f(\cdot)$ is a function that is increasing in $\rho_{i k}$, i.e., better PSR at $k$ implies higher benefit, and decreasing in $\rho_{i m}$, i.e., a bad PSR at a macro-station $m$ implies that SCBS $k$ gets more benefit by offloading $i$ from the macro-cell network. Although the analysis in the rest of this paper applies to any function $f(\cdot)$, we use the following function which represents a cell range expansion-oriented metric:

$$
h_{k}(i)=\beta \frac{\rho_{i k}}{\rho_{i m}},
$$

with $\beta$ a price per PSR ratio that allows the operator to control the "bias" for offloading traffic. Clearly, the utility in (6) is in line with the recent efforts on cell biasing/range expansion [12], [20] and presents an interesting way to control and quantify this bias.

Finally, the benefit that any macro-station $m \in \mathcal{M}$ extracts from a user $i \in \mathcal{N}$ is simply an increasing function of the PSR achieved by $i$ at $m$ as follows:

$$
w_{m}(i)=\rho_{i m} .
$$

Having laid down the main components of the studied model, in the next section, we formulate the access point assignment problem as a college admissions game.

\section{A College Admissions Game for Access Point SELECTION}

\section{A. Game Formulation and Preferences}

To formally model the studied problem, we use the framework of college admissions games, also known as many-to-one matching games [21], [22]. The college admissions problem, as introduced in [21], models the interactions between a number of students wishing to apply to a number of colleges, each of which has a fixed quota on the number of students that it can admit. Given often conflicting preferences between the students and the colleges, it is of interest to study how one can assign the students to colleges while satisfying, as much as possible, all preferences [21], [22]. This framework is suitable for the 
proposed problem as it allows capture of the heterogeneous nature of a small cell network while characterizing stable and desirable association points. Within the studied small cell network, we formulate a college admissions game, referred to as the small cell admissions game, defined by three components: 1) the set $\mathcal{N}$ of wireless users acting a students, 2) the set $\mathcal{A}$ of access points acting as colleges, each access point having a certain quota on the maximum number of users that it can admit, and, 3) preference relations for the access points and users allowing them to build preferences over one another. The solution of this game is an assignment between the users and the access points that satisfies their preferences and constraints. We consider that all players (users, SCBS, macro-station) are honest nodes that do not cheat during the association process. The case in which one or more nodes are cheating can constitute an interesting topic for future work that is out of the scope of the current paper. Here, we note that matching games have been recently used in basic wireless resource allocation [23], [24] and cognitive radio [25]. However, unlike the work done here, these existing approaches do not deal with the specifics of small cell networks and are focused mostly on one-to-one matching. Moreover, they do not explore the idea of college transfers, needed to handle the coupling between users' strategies.

Due to its low cost nature, any $\operatorname{SCBS} k \in \mathcal{K}$ can service only a maximum number (quota) $q_{k}$ of users (typically a small value [1]). For the macro-stations, although no physical constraints impose a maximum quota, each macro-station $m \in$ $\mathcal{M}$ sets a certain quota of users $q_{m}$ that it is willing to accept. Specifically, in order to ensure that every available user will eventually be serviced, each macro-station $m \in \mathcal{M}$ chooses its quota $q_{m}$ to be equal to the maximum number of users that will potentially connect to $m$, in the absence of SCBSs and based on a standard assignment in which each user chooses its closest macro-station.

A preference relation $\succeq_{i}$ for a college (student) is defined as a complete, reflexive, and transitive binary relation over the set of all students (colleges) ${ }^{3}$. Using these preferences, the access points and the users can rank one another. For an SCBS $k \in \mathcal{K}$, we define a preference relation $\succeq_{k}$ over the set of users $\mathcal{N}$, such that, for any two users $i, j \in \mathcal{N}, i \neq j$, we have

$$
i \succeq_{k} j \Leftrightarrow h_{k}(i) \geq h_{k}(j)
$$

where $h_{k}(\cdot)$ is given by (6). In other words, the SCBSs can rank their users by giving a preference to the ones that generate a higher benefit as per (6). For any macro-station $m \in \mathcal{M}$, a preference relation $\succeq_{m}$ over the set of users $\mathcal{N}$, is defined as follows, for any two users $i, j \in \mathcal{N}, i \neq j$ :

$$
i \succeq_{m} j \Leftrightarrow w_{m}(i) \geq w_{m}(j)
$$

where $w_{m}(\cdot)$ is given by (7). Thus, the macro-stations simply rank the users based on the PSR. Note that, in a college admissions game it is often desirable to have strict preferences, denoted by $\succ$, and, thus, hereinafter, we consider that whenever any player is indifferent between two choices, it will randomly rank one before the other (e.g., toss a coin).

While for the access points, one can build preferences based on the utilities in (6) and (7), dealing with the users' preferences introduces some complications. In particular, the R-factor of the

${ }^{3}$ Variants of this game allow the colleges to build preferences over subsets of students [22]. However, in wireless networks, such a model would be undesirable since its complexity for building preferences grows exponentially with the network size as per the Bell number [22]. users as in (4) depends on the number of other users admitted at a given access point through the delay component. Thus, if one defines a preference relation for the users based on (4), this relation will depend on the choices of the other users, due to the interdependence in the delay. In this respect, most literature that deals with the college admissions game [21], [22] assumes that the preferences of the students do not depend on other students' choices. Although such an assumption is reasonable within game theory, it does not hold in the proposed small cells game. As discussed in [22], when dealing with interdependent preferences, finding acceptable solutions for the general college admissions game becomes complex. Even though putting some restrictions on the preferences can make these solutions more tractable, the additional constraints often require computing preferences over all subsets of the users or access points [22], which yields an exponential complexity that is unsuitable for wireless communication problems in which the number of users can grow significantly.

To overcome this challenge, we divide our proposed game into two interdependent subgames: a small cell admission subgame with R-factor guarantees and an access point transfers subgame. In the first subgame, the users build their preferences based on the potential R-factor guarantees that each access point can guarantee. Based on the results of the first subgame, in the second subgame, some of the users can request to transfer to other access points (perform a college transfer) if possible (e.g., if some access points did not fill up their entire quotas).

\section{B. Admissions Game with Guarantees}

In the first subgame, the users build their preferences based on the R-factor level that each access point $a \in \mathcal{A}$ can guarantee. This R-factor guarantee depends on the maximum delay that is experienced at $a$. Since, within a certain time period, each access point is aware of an estimate of its backhaul delay, the maximum delay $\bar{\tau}_{a}$ in (2) at an access point $a \in \mathcal{A}$ depends on the maximum wireless access delay $\bar{\tau}_{a}^{\mathrm{w}}$ given by

$$
\bar{\tau}_{a}^{\mathrm{w}}=\left(q_{a}-1\right) \cdot \theta,
$$

where $q_{a}$ is the quota of access point $a$. As a result, the maximum delay at an access point $a$ is simply $\bar{\tau}_{a}=\bar{\tau}_{a}^{\mathrm{W}}+\tau_{a}^{\mathrm{b}}$. In consequence, based on the maximum potential delay, every user $i \in \mathcal{N}$, can define a preference relation $\succeq_{i}$ such that, for any two access points $a, b \in \mathcal{A}, a \neq b$

$$
a \succeq_{i} b \Leftrightarrow u_{i}(a) \geq u_{i}(b),
$$

where $u_{i}(a)=U_{i}\left(\rho_{i a}, \bar{\tau}_{a}\right)$, with $U_{i}\left(\rho_{i a}, \bar{\tau}_{a}\right)$ as in (4), is a preference function that assigns for each access point $a$ the $\mathrm{R}$-factor in (4) that $a$ guarantees for user $i$.

Using the preferences in (8), (9), and (11), we can define the first subgame as a college admissions problem with R-factor guarantees. The solution of this subgame is a matching $\mu$ which is a mapping defined on the set $\mathcal{A} \cup \mathcal{N}$ and satisfying, for all access point $a \in \mathcal{A}$ and user $i \in \mathcal{N}, 1) \mu(a) \in 2^{\mathcal{N}}$, and, 2) $\mu(i) \in \mathcal{A} \times \mathfrak{B}_{i} \cup\{\emptyset, \emptyset\}$, where $2^{\mathcal{N}}$ is the set of all subsets of $\mathcal{N}$ and $\mathfrak{B}_{i}$ is the set of all subsets of $\mathcal{N}$ that contain user $i$. In other words, $\mu$ is a mapping that assigns for every access point a subset of users and for every user an access point. Alternatively, the solution of this subgame can be seen as a partition $\Pi$ of the set $\mathcal{N}$ such that $\Pi=\left\{S_{1}, \ldots, S_{M+K}\right\}, S_{a} \cap S_{b}=\emptyset, a \neq$ $b, \cup_{l=1}^{M+K} S_{l}=\mathcal{N}$ and each $S_{a} \subseteq \mathcal{N}$ is a coalition of users that are using access point $a$. If, for a given $l \in\{1, \ldots, M+$ $K\}, S_{l}=\emptyset$, then access point $l$ has no users assigned to it. 


\section{A Coalitional Game for College Transfers}

Upon learning of the partition $\Pi$ resulting from the small cell admissions game with guarantees, the access points and the users might have an incentive to negotiate potential user transfers, depending on the actual perceived R-factor as per (4). For example, if some access points within $\Pi$ have used very small fractions of their quotas, it can be beneficial to transfer some users from highly loaded access points to the lightly loaded access points. To study these inter-access point transfers, we use the framework of coalitional game theory [26]. Formally, we define a coalitional game among the users that is identified by the pair $(\mathcal{N}, V)$ where $\mathcal{N}$ is the set of players, i.e., users, and $V$ is a mapping that assigns for every coalition $S_{a} \subseteq \mathcal{N}$, formed around an access point $a$, a payoff vector $\boldsymbol{U}$ in which each element $U_{i}\left(\rho_{i a}, \tau_{a}\right)$ is the R-factor of user $i$ as per (4). Here, each coalition $S_{a} \subseteq \mathcal{N}$ represents a group of users that are connected to an access point $a \in \mathcal{A}$.

In this $(\mathcal{N}, V)$ coalitional game, one can see that the grand coalition, i.e., all users forming a single coalition at a certain access point, can never form due to the increased delay and the fact that no access point can accommodate all the users in the network. Instead, disjoint coalitions, each of which is centered at a given access point, need to form. As a result, this game is classified as a coalition formation game [26] in which the objective is to enable the users to change from one coalition to another, depending on their utilities, the acceptance of the access points, and the different quotas. In order to achieve this objective, for every user $i$, we formally define the following transfer rule:

Definition 1: Transfer Rule - Consider any partition $\Pi=$ $\left\{S_{1}, \ldots, S_{M+K}\right\}$ of $\mathcal{N}$, where $S_{a}$ is a coalition served by an access point $a \in \mathcal{A}$. A user $i$ has an incentive to transfer from its current coalition $S_{a}$, for some $a \in\{1, \ldots, M+K\}$ and join another coalition $S_{b} \in \Pi, S_{a} \neq S_{b}$, if: 1) user $i$ improves its R-factor in (4) by transferring, i.e., $U_{i}\left(\rho_{i b}, \tau_{b}\right)>U_{i}\left(\rho_{i a}, \tau_{a}\right)$, and, 2) access points $a$ and $b$ approve of the transfer.

The acceptance of a transfer by the access points is dependent upon their quotas and their willingness to allow the transfer. A pair of access points $a, b \in \mathcal{A}$ are willing to accept the transfer of a user $i$ from $a$ to $b$, if:

1) Access point $b$ that is serving the users in $S_{b}$ and which will potentially accept the transfer $S_{b}$ does not exceed its quota $q_{b}$, i.e., $\left|S_{b} \cup\{i\}\right| \leq q_{b}$.

2) The social welfare, i.e., the overall R-factor, perceived at both access points $a$ and $b$ is increased. Thus, we have $v\left(S_{a} \backslash\{i\}\right)+v\left(S_{b} \cup\{i\}\right)>v\left(S_{a}\right)+v\left(S_{b}\right)$, where we define $v\left(S_{a}\right)=\sum_{i \in S_{a}} U_{i}\left(\rho_{i a}, \tau_{a}\right)$ as the total utility generated by a coalition $S_{a}$.

The motivation behind this acceptance rule is two-fold: 1) it provides a way for the access points to participate and have some control on the users coalitional game, and, 2) it enables a collaboration between the access points that values the overall social welfare of the network. Given that the coalitional transfers game occurs after the first subgame, the transfer rule and its corresponding acceptance criteria, enable the access points to maintain some control over their initially preferred and accepted users' list. At this stage, any pair of access points $a$ and $b$ that are involved in a transfer have an incentive to be cooperative, i.e., to accept the transfer only in agreement, since, in the future, they can be involved in other transfers in an opposite direction, and, hence, require their peers' potential future cooperation.

\section{Game Solution And Algorithm}

Thus far, we have modeled the access point game as a college admissions game with transfers and decomposed it into two subgames: an admissions game with R-factor guarantees and a coalitional transfers game. As a solution, we propose an algorithm, shown in Algorithm 1, having two main phases, each of which solves a corresponding subgame.

First subgame solution: For the first subgame, a suitable solution would be a stable and optimal matching $\mu^{*}$, defined as follows [21]:

Definition 2: A user-access point matching $\mu^{*}$ is said to be stable, if there does not exist any pair of users $i, j \in \mathcal{N}$ that are assigned (using $\mu^{*}$ ), respectively, to access points $a, b \in \mathcal{A}$, although $j$ prefers $a$ to $b$, i.e., $a \succ_{j} b$ and $a$ prefers $j$ to $i$, i.e., $j \succ_{a} i$. Further, a matching $\mu^{*}$ is said to be optimal, if every user is at least as well off under it as under any other stable assignment.

To find such a stable and optimal matching for our first subgame, we use the Gale and Shapley deferred acceptance method, first introduced in [21], and shown to reach a stable and optimal matching between the colleges and the students. The deferred acceptance method is described in details in Phase I of Algorithm 1. In essence, after ranking one another using the preferences in (8), (9), and (11), the users start by submitting their applications for assignment to their top preferred access point. Then, each access point $a \in \mathcal{A}$ receives the requests, e.g., over a control channel, and places the top ranked $q_{a}$ users/applicants on a waiting list, while rejecting the rest. The procedure repeats as the rejected applicants submit requests for admission to their next preferred access point. Again, each access point $a$ places, on a new waiting list, the top ranked $q_{a}$ users among the previous waiting list and the new applicants, and rejects the rest. This procedure is repeated and converges to a stable and optimal matching $\mu^{*}$ once every applicant in $\mathcal{N}$ is placed on a certain waiting list [21] (or has been rejected from every access point, but, this case is unlikely to happen in the proposed model since the macro-stations' quotas can fit all users, if adequate resources are available) in which case the access points make their initial admissions lists as per the matching $\mu^{*}$, which constitutes the solution to the first subgame.

The matching $\mu^{*}$ resulting from the deferred acceptance method is stable and optimal for networks in which the users are mainly interested in guaranteeing a certain R-factor level, as per (11). One example is a network in which the users are interested in voice messaging services or interactive voice response (IVR) services in which the service quality is not sensitive to variations in the R-factor above a certain guaranteed value. In such networks, it is possible to consider the matching $\mu^{*}$ as a suitable solution for the overall admissions game.

Second subgame solution: As soon as Phase I converges to a matching $\mu^{*}$ equivalent to a partition $\Pi_{\mathrm{DA}}=$ $\left\{S_{1}, \ldots, S_{M+K}\right\}$ of $\mathcal{N}$, the access points open a window for transfers to the users, which indicates the start of the second subgame. Basically, the second subgame enables the users to explore possibilities for improving their R-factors by transferring to other access points, within the matching $\mu^{*}$, 


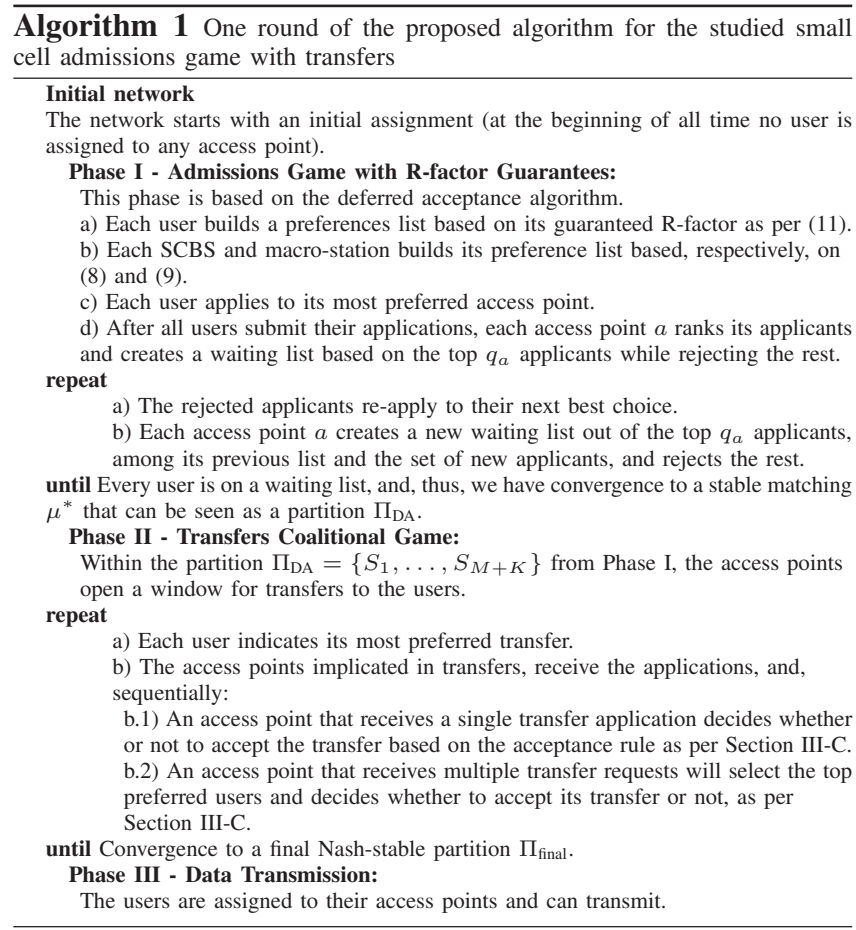

based on the actual R-factor in (4). The need for the transfers is primarily important in networks in which the outcome of the deferred acceptance method does not fill all (or a significant part of) the quotas of the SCBSs and macro-stations (e.g., medium-sized networks). Inherently, the transfers coalitional game enables a better load balance at the access points and improves the performance of the network and users.

For solving the second subgame, we propose a coalition formation algorithm based on the transfer rule as described in details in Phase II of Algorithm 1. In this phase, starting with the partition $\Pi_{\mathrm{DA}}$ resulting from the deferred acceptance method, each user makes its most preferred transfer request, i.e., the transfer that increases most its R-factor as per (4). The transfer requests are then received by the access points and, based on (8) and (9), each access point $a \in \mathcal{A}$ chooses its most preferred applicant $i$ wishing to transfer from another access point $b$. Access points $a$ and $b$ negotiate, in a pairwise manner, whether or not the transfer can be accepted given the current partition, i.e., the quota of $a$ is not exceeded and the overall utility of the two involved access points increases. If the acceptance conditions are satisfied, then $a$ and $b$ agree on the transfer of $i$. In this process, the acceptance of the transfers occurs at the level of the access points, sequentially, in any arbitrary order. The process is repeated until convergence, which is guaranteed, as follows:

Theorem 1: Starting from any initial partition $\Pi_{\mathrm{DA}}$, the proposed coalition formation algorithm for transfers is guaranteed to converge to a final partition $\Pi_{\text {final }}$.

Proof: Given a partition $\Pi_{\mathrm{DA}}$ resulting from the deferred acceptance phase of Algorithm 1, the coalition formation process can be seen as a sequence of transfer operations that transform the network's partition, e.g.,

$$
\Pi_{0}=\Pi_{\mathrm{DA}} \rightarrow \Pi_{1} \rightarrow \Pi_{2} \rightarrow \ldots,
$$

where $\Pi_{l}=\left\{S_{1}, \ldots, S_{M+K}\right\}$ is a partition, composed of at most $M+K$ coalitions (since each coalition forms at a given access point) that is formed after $l$ transfers. Recall that every transfer operation from an access point $a$ to an access point $b$ yields

$$
v\left(S_{a} \backslash\{i\}\right)+v\left(S_{b} \cup\{i\}\right)>v\left(S_{a}\right)+v\left(S_{b}\right) .
$$

As a transfer between two access points $a$ and $b$ in a partition $\Pi_{l}$, does not affect the total utility generated by coalitions in $\Pi_{l} \backslash$ $\left\{S_{a}, S_{b}\right\}$ (since advanced interference management techniques such as in [4], [16], and [17] are used), then, every transfer $\Pi_{l} \rightarrow \Pi_{k}$, forms an order such that

$$
\Pi_{k} \rightarrow \Pi_{l} \Leftrightarrow \sum_{S_{m} \in \Pi_{k}} v\left(S_{m}\right)>\sum_{S_{p} \in \Pi_{l}} v\left(S_{p}\right)
$$

which is transitive and irreflexive. Therefore, for any two partitions in the sequence (12), we have $\Pi_{l} \neq \Pi_{k}, l \neq k$. As the number of partitions of a set is finite and equal to the Bell number [22], then, the sequence in (12) is guaranteed to converge to a final partition $\Pi_{\text {final }}$.

Partition $\Pi_{\text {final }}$ is the final access point assignment, after which the users start their data transmission in the last phase of Algorithm 1. Although the partition $\Pi_{\text {final }}$ results from the transformation of a stable matching $\mu^{*}$, it is difficult to guarantee analytically that this stability is preserved within $\Pi_{\text {final }}$ after transfers. Intuitively, one can see that, whenever the number of transfers is small, such as in a dense network, the stability of the matching will be maintained. Nevertheless, we can guarantee a weaker for of stability for $\Pi_{\text {final }}$ [26]:

Definition 3: A partition $\Pi=\left\{S_{1}, \ldots, S_{M+K}\right\}$ of a players set $\mathcal{N}$ is Nash-stable if for every user $i \in \mathcal{N}, i \in S_{a}, S_{a} \in \Pi$, having a preference relation $\triangleright_{i}$ over its possible coalitions at access points in $\mathcal{A}$, we have $\forall i \in \mathcal{N}, S_{a} \succeq_{i} S_{b} \cup\{i\}$ for all $S_{b} \in \Pi \cup\{\emptyset\}, i \notin S_{b}$.

A coalition partition $\Pi$ is Nash-stable, if no user has an incentive to move from its current coalition to another coalition in $\Pi$ based on a well-defined preference order $\triangleright_{i}$. The transfer operation as per Definition 1, can be mapped into such a preference order $\triangleright_{i}$, such that, for any user $i$, a coalition $S_{a}, i \in S_{a}$ is preferred over another coalition $S_{b} \cup\{i\}$, i.e., $S_{a} \triangleright_{i} S_{b}$ if $U_{i}\left(\rho_{i b}, \tau_{b}\right)>U_{i}\left(\rho_{i a}, \tau_{a}\right),\left|S_{b}\right| \leq q_{b}$, and $v\left(S_{a} \backslash\{i\}\right)+v\left(S_{b} \cup\{i\}\right)>v\left(S_{a}\right)+v\left(S_{b}\right)$. With this definition, a Nash-stable partition becomes a partition in which no user has an incentive to execute a transfer rule, and, consequently, we can state the following lemma:

Lemma 1: Starting from any initial assignment (including the case in which no user is assigned to any access point), Algorithm 1 always converges to a Nash-stable partition in the preference order $\triangleright_{i}$.

Proof: First, Phases I and II of Algorithm 1 are guaranteed to converge as per [21] and Theorem 1, respectively. Assume that a partition $\Pi_{\text {final }}$ resulting from Algorithm 1 is not Nashstable; then there exists a user $i \in S_{a}, S_{a} \in \Pi_{\text {final }}$ and a coalition $S_{b} \in \Pi_{\text {final }}$, such that $S_{b} \cup\{i\} \triangleright_{i} S_{a}$, and user $i$ can transfer from $S_{a}$ to $S_{b}$ (given the approval of the SCBSs). However, this contradicts the convergence result of Theorem 1 and, thus, $\Pi_{\text {final }}$ must be Nash-stable.

In a practical network, to implement Algorithm 1, each macro-station or SCBS will broadcast a pilot signal over a control channel that contains information on its delay guarantees and will discover the users using any common technique used in cellular networks [14]. Meanwhile, the users will monitor the pilot channel, obtain the delay information and estimate the PSR to each access point, using standard signal processing 


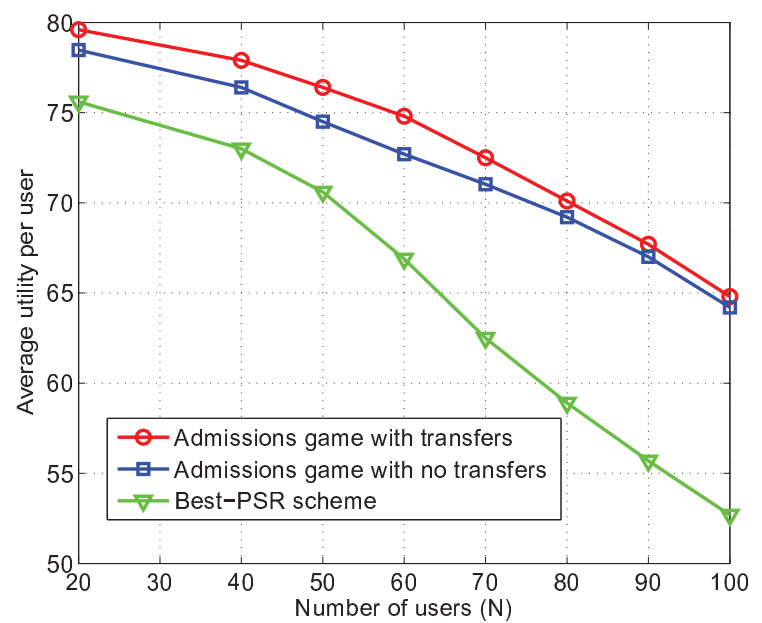

Fig. 2. Performance, in terms of average utility per user (i.e., R-factor as per (4)), as the number of users $N$ increases for a network with $K=10$ SCBSs.

techniques. The use of such pilot signals for user-to-access-point interaction is common in many standard wireless systems [1]. Then, the users and access points can build their preferences based on (8), (9), and (11). Subsequently, the users and access points engage in the deferred acceptance method while signalling their preferences over control channels. The access points can start finalizing their initial waiting once they note an absence of new requests. Then, the access points inform the users of the admitted lists, convey their potential delay using the pilot signal, and open a window for transfer. Afterwards, users that are interested in improving their actual rates investigate potential transfers by monitoring, once again, the pilot signal. Subsequently, the transfer process can be performed in a manner analogous to the deferred acceptance procedure. We note that the transfer process would require pairwise interactions, over the backbone, between involved access points. With regards to complexity, although no known results exist for the deferred acceptance procedure in the general many-to-one case [22], experimental analysis and complexity bounds suggest that the algorithm, in general, has a reasonable complexity which motivates its adoption in several settings [22]. For the transfers algorithm, the complexity lies in the process of identifying transfer operations which, from a user's perspective, has a worst case complexity of $O(M+K)$. However, the users can, in practice, look for potential transfers progressively by exploring some specific network characteristics, e.g., starting with the closest, or with assistance from the access points.

\section{Simulation Results and Analysis}

For simulations, we consider a macro-cell network composed of $M=2$ adjacent cells, with each cell being a square area of $1 \mathrm{~km} \times 1 \mathrm{~km}$ with the macro-station at the center. In this macro-cell network, we randomly deploy the SCBSs and the users. We set all users' transmit powers to $20 \mathrm{~mW}$, the noise plus interference level to an average of $-110 \mathrm{dBm}$ (at all access points), the slot duration to $\theta=20 \mathrm{~ms}$, the propagation loss to $\alpha=3$, the modulation scheme used is BPSK, and $\beta=1$. Unless stated otherwise, the packet size to $B=256$ bits and the quota of the SCBSs is set to a typical value of $q_{k}=4, \forall k \in \mathcal{K}[1]$. The backhaul delay is based on a Pareto distribution with a Pareto index of 1.16 (80-20 rule) which is known to be one suitable delay model for backbone traffic [18]. We select the

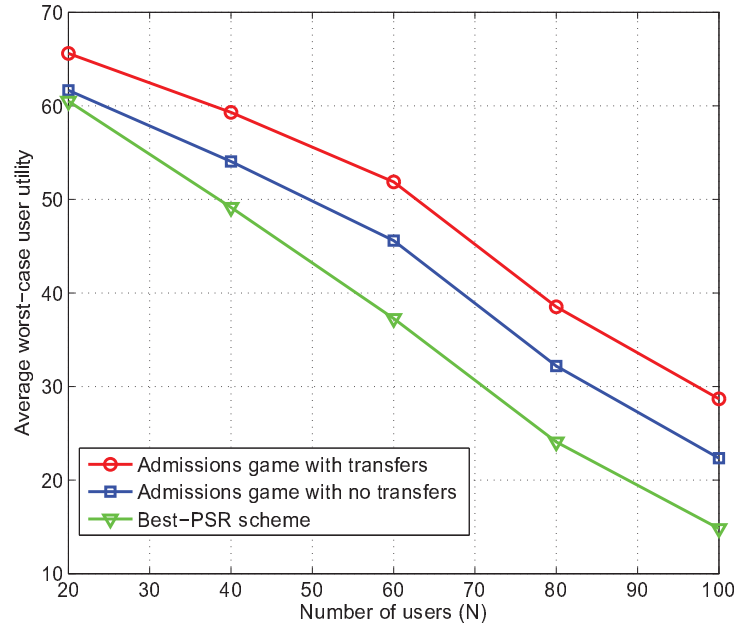

Fig. 3. Worst-case user utility (averaged over random locations of SCBSs and users) for a network with $K=10$ SCBSs as the number of users $N$ varies.

backhaul delay's scale factors of the SCBSs and macro-stations as $40 \mathrm{~ms}$ and $15 \mathrm{~ms}$, respectively. For services, we select the G.729 codec; hence $v_{1}=12, v_{2}=15$ and $v_{3}=0.6$ [19]. All statistical results are averaged, via about 10,000 runs, over possible locations of the SCBSs and users and realizations of the backhaul delay.

In Fig. 2, we show the average utility per user resulting from the proposed small cell admissions game, with and without transfers, for a network with $K=10$ SCBSs, as the number of users $N$ increases. The performance is compared with a bestPSR algorithm that is commonly used in practical networks [1] and in which each user selects the access point with the best PSR while respecting the quotas [1]. Note that this scheme has been selected for comparison purposes since, to the best of our knowledge, this paper is the first in the literature that deals with the assignment problem in the context of uplink small cell networks and our studied model is significantly different from those used in classical cellular networks such as in [13] and [14]. Fig. 2 shows that, as $N$ increases, the performance, i.e., R-factors as per (4), of all three algorithms decreases, due to the increasing delay. Fig. 2 demonstrates that, at all network sizes, the proposed game with and without transfers, yields a performance advantage over the best-PSR algorithm. This advantage reaches up to $23 \%$ of utility improvement relative to the best-PSR algorithm at $N=100$ users. Also, Fig. 2 corroborates the fact that the use of transfers presents an advantage mainly for low-to-medium sized networks, i.e., between $N=40$ and $N=70$. This advantage starts decreasing beyond $N=70$ users, as the quotas start to fill up.

We further assess the performance of the proposed algorithm by showing, in Fig. 3, the average utility achieved by the worst-case user, i.e., cell-edge users, for a network with $K=10$ SCBSs, as the number of users $N$ varies. Fig. 3 shows that, at all values of $N$, the proposed admissions game with and without transfers yields a significant advantage over the best-PSR algorithm. This advantage is increasing with the network size $N$. This is due to the fact that, as more users are deployed, the proposed admissions game handles efficiently the preferences of the users and, hence, yields a performance advantage reaching, for the games with and without transfers, respectively, up to $93.5 \%$ and $50.8 \%$ relative to the best-PSR 


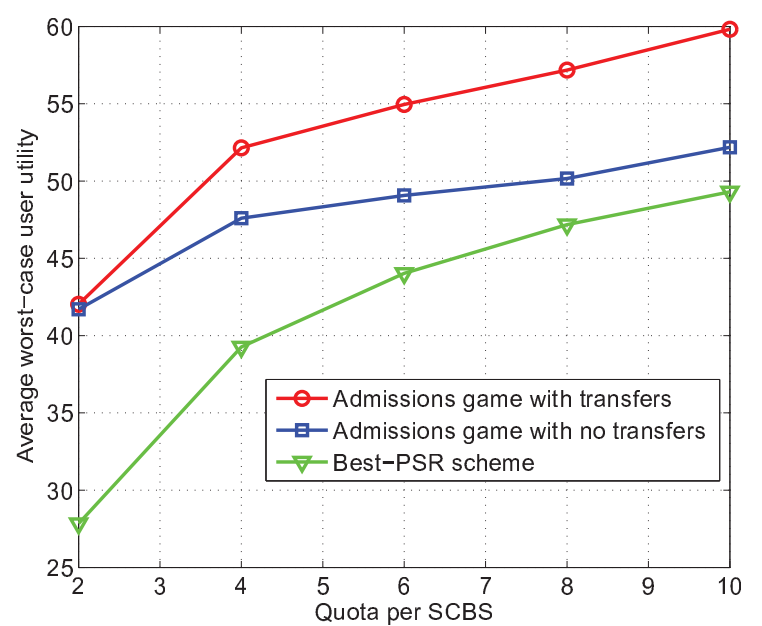

Fig. 4. Worst-case user utility (averaged over random locations of SCBSs and users) for a network with $K=10$ SCBSs and $N=60$ users as the quota per SCBS varies.

algorithm, at $N=100$ users. Furthermore, Fig. 3 demonstrates that the use of college transfers significantly improves the performance of the worst-case user as the proposed game with transfers has a performance advantage increasing with $N$ and reaching up to $28.5 \%$ relative to the game with no transfers. Combining this result with the results of Fig. 2 demonstrates that, as the network size increases, the benefit from using college transfers is more pronounced for the users experiencing poor performance and who are more apt to transfer to another access point, if and when possible.

Fig. 4 shows the achieved average utility for the worstcase user as the quota per SCBS varies for a network with $K=10$ SCBSs and $N=60$ users. In this figure, we can see that, as the quota increases, the average utility for the worst-case user increases for all three schemes. This result is due to the fact that, as the quota increases, the assignment possibilities for the users increase (even in the best-PSR scheme), and, thus, a better utility can be achieved. As the quota becomes larger, the best-PSR algorithm's performance improves; however, it is still outperformed by the proposed admissions game at all quotas. In fact, Fig. 4 demonstrates that, at all quotas, the proposed admissions game with transfers yields a performance advantage between $21.4 \%$ for a quota of 10 users per SCBS and $51 \%$ for a quota of 2 users per SCBS, relative to the best-PSR scheme. The advantage of the proposed game is larger for small quotas which demonstrates the fact that the proposed algorithm is well-adapted to the resource-constrained nature of small cells. Fig. 4 also shows that, as the quota increases, the potential for performing transfers increases, thus improving the advantage of the game with transfers over the game with no transfers. This performance advantage reaches up to $15 \%$ as the quota is around 10 users per SCBS.

Fig. 5 shows, for the proposed game with transfers and the best-PSR scheme, the average number of users that achieve a certain R-factor level for $K=10 \mathrm{SCBSs}$ and $N=60$ users. The histogram of Fig. 5 clearly shows that the small cell admissions game enables the shifting of a significant number of users to better R-factor levels relative to the best-PSR algorithm. For example, compared to the best-PSR scheme, about $22 \%$ of the networks users improve from an "acceptable" service level (range of $] 60-70]$ ) to a "good" service level (range of ]70-80])

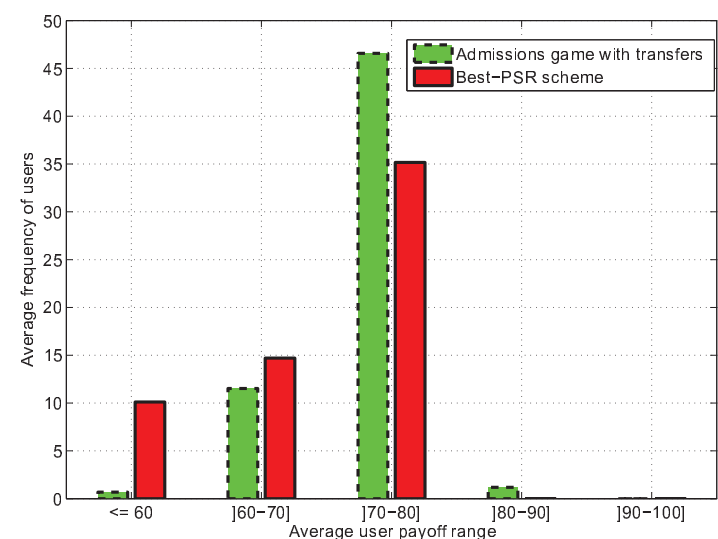

Fig. 5. A histogram on the number of users within each range of average Rfactor (service quality level) for a network with $M=2$ macro-cells, $K=$ 10 SCBSs, and $N=60$ users.

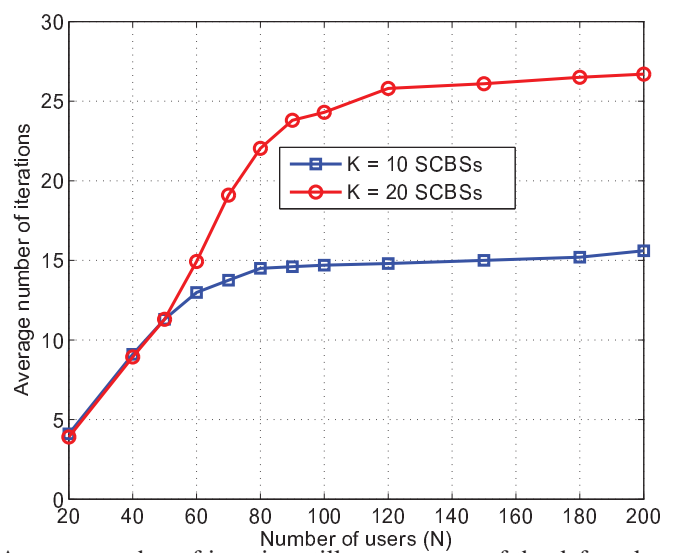

Fig. 6. Average number of iterations till convergence of the deferred acceptance algorithm (Phase I) in Algorithm 1 as the number of users and SCBSs varies.

with a small fraction having a "high" R-factor above 80. Also, while for the best-PSR algorithm, an average of 10 users have a poor service quality (R-factor below 60), using the proposed game, less than 2 users (on the average) experience such poor service. Hence, the proposed game yields, not only average utility gains, but also an improved users' service quality.

Fig. 6 shows the average number of iterations needed for convergence of the deferred acceptance algorithm in Phase I of Algorithm 1 as $N$ and $K$ vary. Fig. 6 shows that, for medium sized networks with $N \leq 50$, the average number of iterations till convergence at $K=10$ and $K=20$ is comparable. But, as $N$ increases above 50, we can see in Fig. 6 that the average number of iterations increases, from about 4 iterations at $N=20$ at all $K$ to around 15.6 and 26.7 , for $K=10$ SCBSs and $K=20$ SCBSs, respectively (at $N=200$ ). In fact, Fig. 6 shows that, as the number of users becomes large relative to the number of SCBSs, i.e., at $N>80$ for $K=10$ SCBSs and at $N>120$ for $K=20$ SCBSs, the average number of iterations becomes almost constant. This is due to the fact that, as $N$ becomes large relative to $M+K$, it is likely that each access point's preferred users would still submit their applications at about the same iteration, hence, not requiring many extra rounds till convergence. Thus, Fig. 6 shows that the deferred acceptance algorithm presents a reasonable convergence time and its complexity grows relatively slowly with $N$ and $K$.

Fig. 7 shows the average utility achieved by each SCBS 


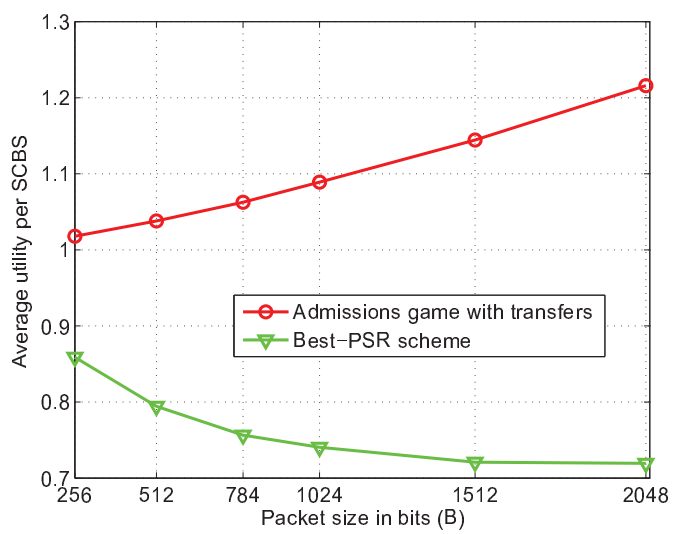

Fig. 7. Performance, in terms of average SCBS utility as per (6), as the packet size $B$ varies for a network with $K=10$ SCBSs and $N=100$ users as per (6) as the packet size $B$ varies for a network with $K=10$ SCBSs. First, we can seen from Fig. 7 that, as $B$ increases, the average SCBS utility achieved by the proposed game increases while that of the best-PSR algorithm decreases. In essence, as $B$ increases, the possibility of having users experiencing low macro-cell PSRs due to their traffic and distances from their macro-stations becomes large. As a result, the proposed game enables the SCBSs to better offload these users from the macro-station as corroborated by the increase in the utility with $B$ shown in Fig. 7 (recall from (6) that the SCBS utility is a function, not only of the users achieved PSR but also of its potential best PSR with respect to the macrocellular network). In contrast, assignments resulting from the best-PSR algorithm do not account for the SCBSs preferences at all, as intuitively expected. Fig. 7 shows that the proposed game presents a significant performance advantage, in terms of average SCBS utility, increasing with the packet size $B$ and reaching up to $69 \%$ improvement at $B=2048$ bits relative to the best-PSR scheme. In summary, Fig. 7 demonstrates that the proposed game enables, not only the users to improve their preferences, but also the SCBSs' hence ensuring that all involved decision makers satisfy, as much as possible, their preference.

\section{CONCLUSIONS}

In this paper, we have studied the problem of user assignment within a small cell wireless network, given the preferences and capabilities of the three involved nodes: the users, the small cell base stations, and the macro-stations. To do so, we have formulated a college admissions game with transfers in which the access points and the users rank one another, in an effort to reach an agreement over a user-to-access point assignment, given the nodes' preferences. We have defined well-suited preferences that capture the users' need to optimize their packet success rates and delays as well as the SCBSs' incentive to offload traffic from the macro-cell network and extend its coverage. To solve the game, we have proposed a two-phase algorithm: a first phase based on the deferred acceptance algorithm, which finds a stable matching with R-factor guarantees; and a second phase, based on coalition formation games, which enables the users to transfer from one access point to another, when and if possible. We have shown the convergence of the algorithm and have discussed the properties of the resulting partition. Using simulations, we have demonstrated the effectiveness of our proposed college admissions game with transfer. Future work can address a number of interesting open questions and directions, including extending the work to a dynamic-game setting to handle handovers or enabling the users to simultaneously connect to multiple access points (e.g., for diversity) and to account for advanced communication techniques such as cooperative communications or interference alignment.

\section{REFERENCES}

[1] T. Q. S. Quek, G. de la Roche, I. Guvenc, and M. Kountouris, Small Cell Networks: Deployment, PHY Techniques, and Resource Management. Cambridge University Press, 2013.

[2] V. Chandrasekhar, J. G. Andrews, and A. Gatherer, "Femtocell networks: A survey," IEEE Commun. Mag., vol. 46, no. 9, pp. 59-67, Sep. 2008.

[3] D. Lopez-Perez, A. Valcarce, G. de la Roche, and J. Zhang, "OFDMA femtocells: A roadmap on interference avoidance," IEEE Commun. Mag., vol. 47, pp. 41-48, Sep. 2009.

[4] N. Saquib, E. Hossain, L. B. Le, and D. I. Kim, "Interference management in OFDMA femtocell networks: Issues and approaches," IEEE Wireless Commun. Mag., no. 3, pp. 86-95, Jun. 2012.

[5] S. Guruacharya, D. Niyato, E. Hossains, and D. I. Kim, "Hierarchical competition in femtocell-based cellular networks," in Proc. IEEE Global Communication Conference, Miami, FL, USA, Dec. 2010.

[6] C. Xu, L. Song, Z. Han, Q. Zhao, X. Wang, and B. Jiao, "Efficient resource allocation for device-to-device underlaying networks using combinatorial auction," IEEE J. Select. Areas Commun., vol. 31, no. 9, pp. 348-358, Sep. 2013.

[7] F. Liu, E. Erkip, M. Beluri, R. Yang, and E. Bala, "Dual-band femtocell traffic balancing over licensed and unlicensed bands," in Proc. IEEE Int. Conf. on Communications, Workshop on Small Cell Wireless Networks, Ottawa, Canada, Jun. 2012.

[8] I. Guvenc, "Statistics of macrocell-synchronous femtocell-asynchronous users-delays for improved femtocell uplink receiver design," IEEE Communications Letters, vol. 13, no. 4, pp. 239-241, Apr. 2009.

[9] F. Pantisano, K. Ghaboosi, M. Bennis, and M. Latva-Aho, "Interference avoidance via resource scheduling in TDD underlay femtocells," in Proc. of IEEE Symp. Personal, Indoor and Mobile Radio Communications, Istanbul, Turkey, Sep. 2010.

[10] D. Lopez-Perez, X.Chu, and I. Guvenc, "On the expanded region of picocells in heterogeneous networks," IEEE J. Select. Topics Signal Process., vol. 6, no. 3, pp. 281-294, Jun. 2012.

[11] W. C. Cheung, T. Q. S. Quek, and M. Kountouris, "Throughput optimization, spectrum allocation, and access control in two-tier femtocell networks," IEEE J. Select. Areas Commun., vol. 30, no. 3, pp. 561-574, Apr. 2012

[12] Q. Ye, B. Rong, Y. Chen, M. Al-Shalash, C. Caramanis, and J. G. Andrews, "User association for load balancing in heterogeneous cellular networks," IEEE Trans. Wireless Commun., arxiv.org/abs/1205.2833 2012.

[13] D. Niyato and E. Hossain, "Dynamics of network selection in heterogeneous wireless networks: An evolutionary game approach," IEEE Trans. Veh. Technol., vol. 58, no. 4, pp. 2008-2017, May 2009.

[14] J. Lee, R. R. Mazumdar, and N. B. Shroff, "Joint resource allocation and base-station assignment for the downlink in cdma networks," IEEE/ACM Trans. Networking, vol. 14, no. 1, pp. 1-14, Feb. 2006.

[15] J. G. Andrews, H. Claussen, M. Dohler, S. Rangan, and M. Reed, "Femtocells: Past, present, and future," IEEE J. Select. Areas Commun., vol. 30, no. 3, pp. 497-508, Apr. 2012.

[16] S. Rangan, "Femto-macro cellular interference control with subband scheduling and interference cancelation," in Proc. IEEE Global Communication Conference, Miami, FL, USA, Dec. 2010.

[17] S.-M. Cheng, S.-Y. Lien, F.-S. Chu, and K.-C. Chen, "On exploiting cognitive radio to mitigate interference in macro/femto heterogeneous networks," IEEE Wireless Commun. Mag., vol. 18, no. 3, pp. 40-47, Jun. 2011.

[18] P. van Mieghem, Performance Analysis of Communications Networks and Systems. New York, USA: Cambridge University Press, Apr. 2006.

[19] ITU-T Recommendation G.107, "The emodel, a computational model for use in transmission planning," ITU-T, Tech. Rep., Jun. 2002.

[20] A. Damnjanovic, J. Montojo, Y. Wei, T. Ji, T. Luo, M. Vajapeyam, T. Yoo, O. Song, and D. Malladi, "A survey on 3GPP heterogeneous networks," IEEE Wireless Communications, vol. 18, no. 3, pp. 10-21, Jun. 2011.

[21] D. Gale and L. Shapley, "College admissions and the stability of marriage," American Mathematical Monthly, vol. 69, pp. 9-15, 1962.

[22] A. Roth and M. A. O. Sotomayor, Two-Sided Matching: A Study in Game-Theoretic Modeling and Analysis. New York, USA: Cambridge University Press, Mar. 1992.

[23] E. A. Jorswieck, "Stable matchings for resource allocation in wireless networks," in Proc. Int. Conference on Digital Signal Processing, Corfu, Greece, Jul. 2011.

[24] Y. Wu, H. Viswanathan, T. Klein, M. Haner, and R. Calderbank, "Capacity, optimization in networks with heterogeneous radio access technologies," in Proc. IEEE Global Communication Conference, Houston, TX, USA, Dec. 2011.

[25] A. Leshem, E. Zehavi, and Y. Yaffe, "Multichannel opportunistic carrier sensing for stable channel access control in cognitive radio systems," IEEE J. Select. Areas Commun., vol. 30, no. 1, pp. 82-95, Jan. 2012.

[26] Z. Han, D. Niyato, W. Saad, T. Başar, and A. Hjørungnes, Game Theory in Wireless and Communication Networks: Theory, Models and Applications. Cambridge University Press, UK, Oct. 2011. 